\title{
Ondes électromagnétiques, risques et cancers
}

\author{
Electromagnetic Waves, Risks and Cancers
}

\author{
A. Ly \\ (C) Lavoisier SAS 2014
}

Les applications qui découlent des nouvelles technologies façonnent chaque jour davantage notre environnement et modifient en profondeur nos vies tant sociales que professionnelles. Des outils tels que les ordinateurs, les fours à micro-ondes, les téléphones portables, les télévisions, les radiodiffusions, les imageries médicales, les antennes satellites, sont devenus indispensables à nos activités quotidiennes. Ces produits technologiques qui vont marquer durablement ce nouveau millénaire sont-ils sans risques sur la santé humaine et environnementale ? En effet, l'exposition aux champs électromagnétiques suscite des inquiétudes légitimes quant à leur nocivité et leurs propriétés mutagènes et cancérigènes [1-5]. Pourtant, beaucoup de connaissances ont été accumulées sur leur nature et caractéristiques physiques depuis leur découverte en 1888 par l'allemand Heinrich Rudolf Hertz (1857-1894) [6]. Les rayonnements électromagnétiques sont représentés sont la forme d'ondes électromagnétiques dont la propagation se fait aussi bien dans l'air que dans le vide et à la vitesse de la lumière dans ce dernier cas. Nul support matériel n'est nécessaire pour cette propagation. Une onde électromagnétique est un flux d'énergie qui ne transporte pas de matière. Elle est émise lors de la variation simultanée du champ électrique et du champ magnétique. Le spectre électromagnétique est constitué d'une multitude d'ondes dont les différents éléments sont caractérisés par leur longueur d'ondes, leur fréquence et leur quantité d'énergie (Fig. 1) [7,8]. Certaines de ces caractéristiques font que des ondes sont potentiellement délétères pour la santé. Invisibles et imperceptibles, elles peuvent pénétrer la matière vivante et provoquer des chocs thermiques, des lésions cellulaires et des altérations de l'ADN $[4,5,9]$. Les plus dangereuses sont celles qui sont au début du spectre électromagnétique, celles dont les longueurs d'ondes sont les plus courtes. En effet, plus la longueur d'onde est courte plus la fréquence est élevée et plus le rayonnement est énergétique. Ces rayonnements peuvent être ionisants ou radioactifs et avoir des conséquences sanitaires et environnementales très domma-

\footnotetext{
A. Ly $(\bowtie)$

Afrocancer, BP 60751, F-75827 Paris cedex 17, France

e-mail : adamaly@gmail.com
}

geables $[8,10,11]$. Ainsi, il est établi que les rayons gamma, les rayons $\mathrm{X}$ et les ultraviolets induisent des effets cellulaires nuisibles et génétoxiques explicatifs de leur pouvoir cancérigène [10-14]. Seulement, l'imagerie médicale et la médecine nucléaire (imagerie par résonnance magnétique [IRM], scintigraphie, radiographie, tomodensitométrie ...) utilisent ces rayonnements à des doses infratoxiques. De plus, la surexposition des patients à des doses même minimales est proscrite pour éviter les effets cumulatifs des radiations. En revanche, la radiothérapie exploite ces propriétés genotoxiques à des fins curatives avec un contrôle très sécurisé sur les zones irradiées, les doses et les temps d'exposition [15-17].

Si les bénéfices et risques associés à l'utilisation de certaines ondes électromagnétiques sont bien documentés, il demeure des interrogations sur la dangerosité des ondes électromagnétiques dont les fréquences sont comprises entre 9 $\mathrm{KHz}$ et $300 \mathrm{GHz}$. Ces ondes, les radiofréquences, sont générées par les téléphones portables, le Wifi, les antennes relais ou les systèmes d'identification par radiofréquence (RFID) $[1-3,5]$. Les études scientifiques réalisées à ce jour ne sont pas concluantes. Nombre d'entre elles sont contradictoires [18]. En mai 2011, le Centre international de la recherche sur le cancer (CIRC) a classé ces ondes en catégorie 2B c'est à dire « potentiellement cancérigène pour l'Homme ». Cette mesure a été prise à la suite d'une méta-analyse réalisée par un groupe de spécialistes. Cette analyse détaillée des données actuelles de la littérature scientifique et médicale a conclu qu'un risque accru de tumeurs cérébrales (gliomes) peut être associé à l'utilisation intensive des téléphones portables $[3,19]$. Les mécanismes de radiocancérogenèse en jeu restent non élucidés même si on sait que ces ondes sont non ionisantes comme la plupart des ondes du spectre électromagnétique. Ainsi, en absence de consensus et d'explications satisfaisantes et rationnelles sur les effets cancérigènes des ondes électromagnétiques des radiofréquences, le principe de précaution a été recommandé $[5,7,19]$.

Quelques années auparavant, en 2002, le CIRC avait déjà classé dans la catégorie $2 \mathrm{~B}$ (cancérigènes possibles pour l'Homme) les champs électromagnétiques d'extrême basse fréquence (CEM-EBF [-50Hz]) produits par les lignes électriques à très haute tension à cause de leurs rôles soupçonnés 


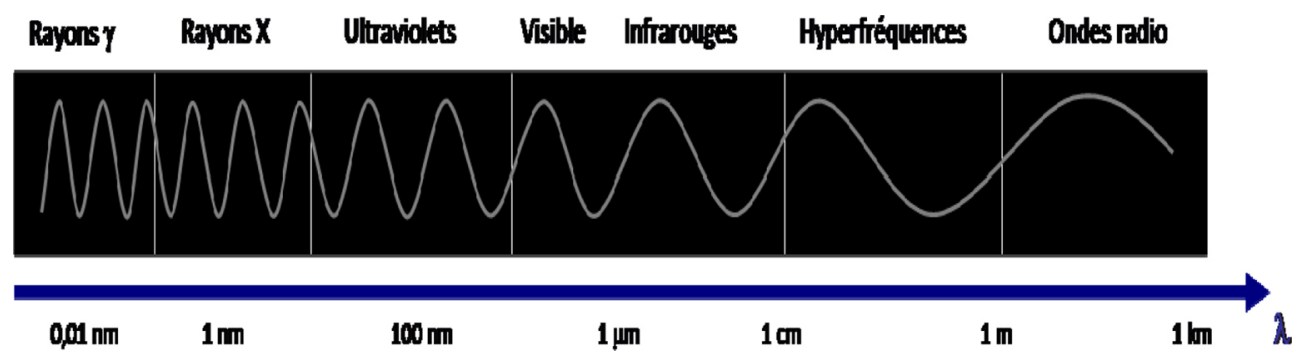

Fig. 1 Spectre des ondes électromagnétiques

par des études épidémiologiques dans l'induction de leucémies infantiles en cas de surexposition [20,20a]. Plus récemment, l'étude CERENAT, une étude cas-témoins multicentrique dont l'objectif était d'évaluer le rôle des téléphones portables dans l'étiologie des tumeurs du système nerveux central (gliome et méningiomes) a été menée par une équipe bordelaise. Elle conclut en l'absence d'association entre la survenue de tumeurs cérébrales et l'exposition aux radiofréquences émises par les téléphones portables. Toutefois, un lien de causalité, statistiquement significatif, a été trouvé en cas de surexposition à ces ondes et l'émergence de gliomes et de méningiomes : 15h/mois d'utilisation de téléphone portable pendant une période médiane de 5 ans multiplient les risques de gliome par 3 et de méningiome par 2,5. Les résultats de cette étude sont donc conformes aux conclusions d'études précédentes sur les radiofréquences comme facteurs étiologiques potentiels de cancers [21].

Par ailleurs, une vaste étude internationale, l'étude « interphone », a abouti à des résultats discordants. Cependant, la tendance est plus marquée vers l'absence d'association entre l'utilisation des téléphones portables et les tumeurs de croissance rapide comme les gliomes. Pour les tumeurs à croissance lente, le période d'observation courte introduit des incertitudes sur l'absence de liaison décrite par certaines équipes. Mais une utilisation intensive de téléphone portable, c'est à dire plus de $30 \mathrm{mn} /$ jour pendant $10 \mathrm{ans}$, augmente de $40 \%$ les risques de developper un gliome [22-24]. En résumé, des recherches expérimentales aux méthodes agrégatives de synthèse des données, l'état des lieux des connaissances montre une insuffisance à la fois quantitative et qualitative doublée d'une incohérence des résultats [2,5,23-25,29]. Malgré des limites méthodologiques et des biais, les diverses études sur les effets sanitaires des ondes électromagnétiques ont joué ce rôle d'alerte si primordial en prévention et gestions des risques de santé.

S'agissant des tumeurs cérébrales, bénignes ou malignes, leur localisation intracrânienne en fait des tumeurs de découverte tardive, ce qui aggrave leur pronostic [30]. Au plan mondial, les cancers du système nerveux central (SNC) ont une incidence, tous sexes confondus, estimée à 3,4 cas pour 100000 habitants avec une mortalité de 2,5 cas/100 000 habitants. Ce sont des cancers rares qui représentent 1,8\%
(256 213 cas) des 14,1 millions de nouveaux cas enregistrés en 2012 [31]. Les gliomes qui se développent aux dépens de la glie, tissu de soutien des neurones, en sont les plus fréquents. Ils se situent au premier rang des tumeurs primitives du cerveau. L'adulte jeune entre 50 et 60 ans est le plus fréquemment atteint et les hommes plus souvent que les femmes. En Afrique subsaharienne, ces types de cancers sont aussi peu fréquents $(0,8$ cas $/ 100000$ habitants en incidence et $0,7 \mathrm{cas} / 100000$ habitants en mortalité). Mais, parmi les 4989 cas de cancers du SNC déclarés, 4056 en sont morts [31,31a]. On cite dans les causes de gliome une susceptibilité/prédisposition génétique liée aux mutations de certains gènes : CCDC26 (coiled-coil domain containing prtotein26), CDKN2B (Cyclin-dependent kinase 4 inhibitor B), TERT, (Telomerase reverse transcriptase, Regulator of Telomere Elongation Helicase 1), PHLDB1 (pleckstrin homology-1 ike domain, family B member 1 ) et IDH1/2 (isocitrate déshydrognénase de type 1/2) [32-35]. Des maladies génétiques rares à transmission autosomique dominante telles que le type I de la neurofibromatose (due à la mutation du gène NF1) et le syndrome de Li-Fraumi (dû à la mutation du gène TP 53) confèrent aussi une susceptibilité accrue aux gliomes [30]. Outre les gliomes, d'autres tumeurs du système nerveux central comme les méningiomes et les neurinomes acoustiques sont parmi les éventuels risques associés à la surexposition aux radiofréquences [26-28] (Figs 2, 3, 4).

Acquérir des informations complémentaires pour dicter les comportements à adopter afin de calmer les peurs injustifiées et les psychoses sont les prochaines étapes dans la compréhension de tous types de risques d'origine électromagnétique. La problématique centrale reste, bien sûr, la carcinogénicité possible de leurs effets suite à des expositions continues ou intermittentes.

De tels outils décisionnels, à même d'orienter des stratégies de santé publique, ne seront fiables et acceptés que s'ils sont fondés sur des arguments scientifiques qui sont sinon irréfutables du moins consensuels et non cacophoniques. En attendant, il est recommandé de limiter la durée des communications et d'utiliser des oreillettes pour réduire l'exposition de la tête aux ondes électromagnétiques. En effet, 5 milliards d'individus à l'échelle de la planète sont équipés de téléphones portables et, de ce fait, soumis aux risques induits. Quant 


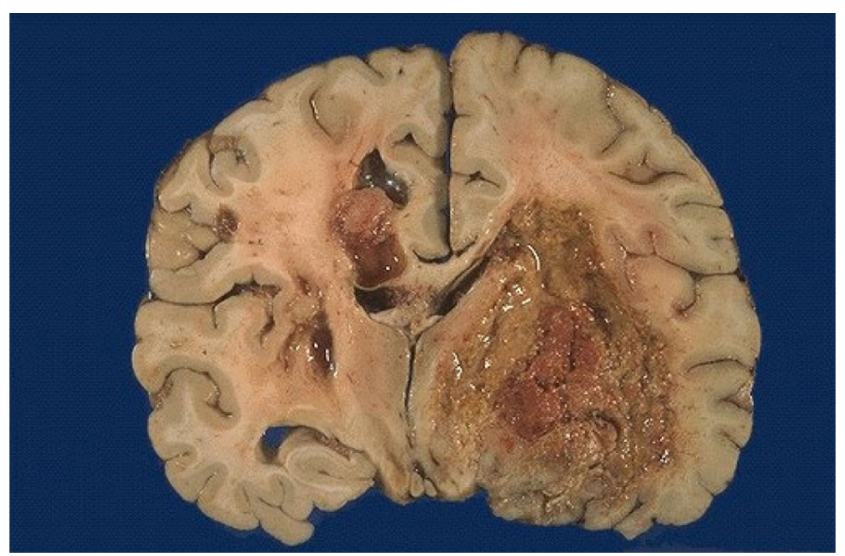

Fig. 2 Coupe axiale d'un cerveau humain montrant une tumeur maligne (glioblastome multiforme) [43]

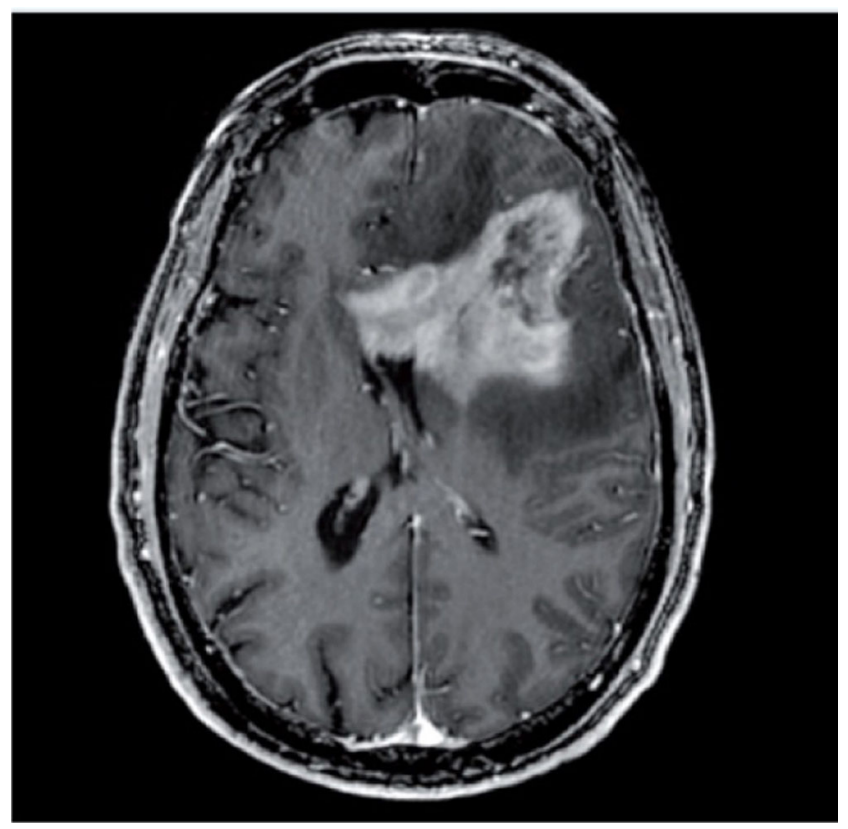

Fig. 3 Imagerie par résonnance magnétique (IRM) de glioblastome de haut grade [44]

à l'Afrique, elle enregistre une explosion de l'usage des téléphones portables avec prés de 620 millions d'abonnés à la fin de l'année 2011 pour une population dépassant désormais le milliard d'habitants (Fig. 5) [36-38]. À ces enjeux de santé se greffent des aspects socio-économiques tellement cruciaux pour le développement du continent. Selon l'union internationale des télécommunications, le secteur de la téléphonie mobile y a généré un chiffre d'affaires de 56 milliards de dollards US et a favorisé la création de 3,5 millions d'emplois directs et indirects au cours de l'année 2010 [36-38]. En définitive, la prise en charge des risques potentiels causés par ces nouvelles technologies doit être globale, responsable et éthique. Pour ce faire, elle exige l'association de toutes les parties prenantes (fabricants, commerciaux, utilisateurs, pro-

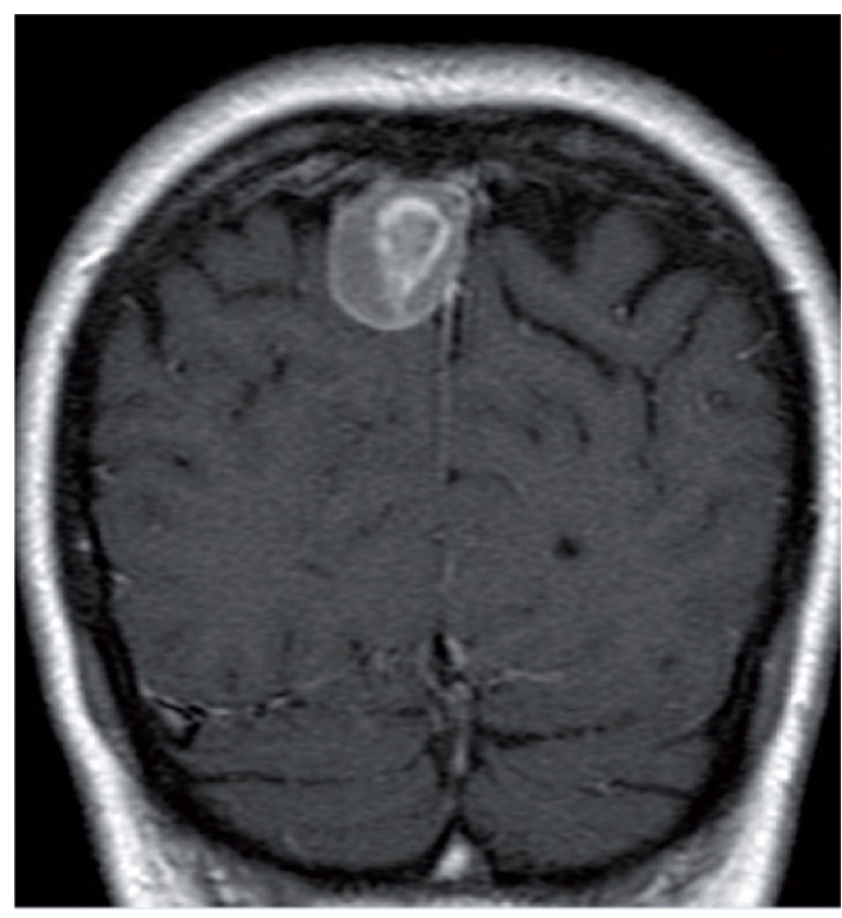

Fig. 4 IRM de Méningiome [44]

fessionnels de santé, législateurs...). Cette révolution technologique et sociétale ne peut exclure la santé, un allié objectif avec qui elle a des intérêts communs et convergents : le bien être des populations.

À bien des égards, ces questions sur l'impact sanitaire des produits innovants ne manqueront pas de se poser de façon récurrente. Des informations appropriées et circonstanciées sur leur innocuité seront des demandes constantes des populations dans les années qui viennent et ce à mesure que des percées technologiques ou biologiques seront opérées. À titre d'exemple, les nanotechnologies, les produits du génie génétique (organismes génétiquement modifiés [OGM], nouveaux vaccins...), les produits alimentaires, les nouveaux procédés de stérilisation ou de fabrication, font naitre des questions, craintes et polémiques relatives à la sécurité sanitaire et environnementale d'où les mobilisations de masse observées [39-41].

Cette méfiance et défiance vis-à-vis des innovations posent la question de la responsabilité sociale, politique ou scientifique des risques encourus au niveau individuel ou collectif à court, moyen et long termes. Puisque le risque zéro relève de l'utopie et que le risque est inhérent aux progrès scientifiques et techniques, le principe de précaution peut être une réponse s'il ne devient une norme abusive au point de freiner toute velléité de recherches ou d'inventions.

En réalité, le principe de précaution s'adresse aux risques probables. Ceux dont l'identification ou la quantification est en attente de validation scientifique. Sa seule finalité est l'évitement des conséquences indésirables. Quant à la 


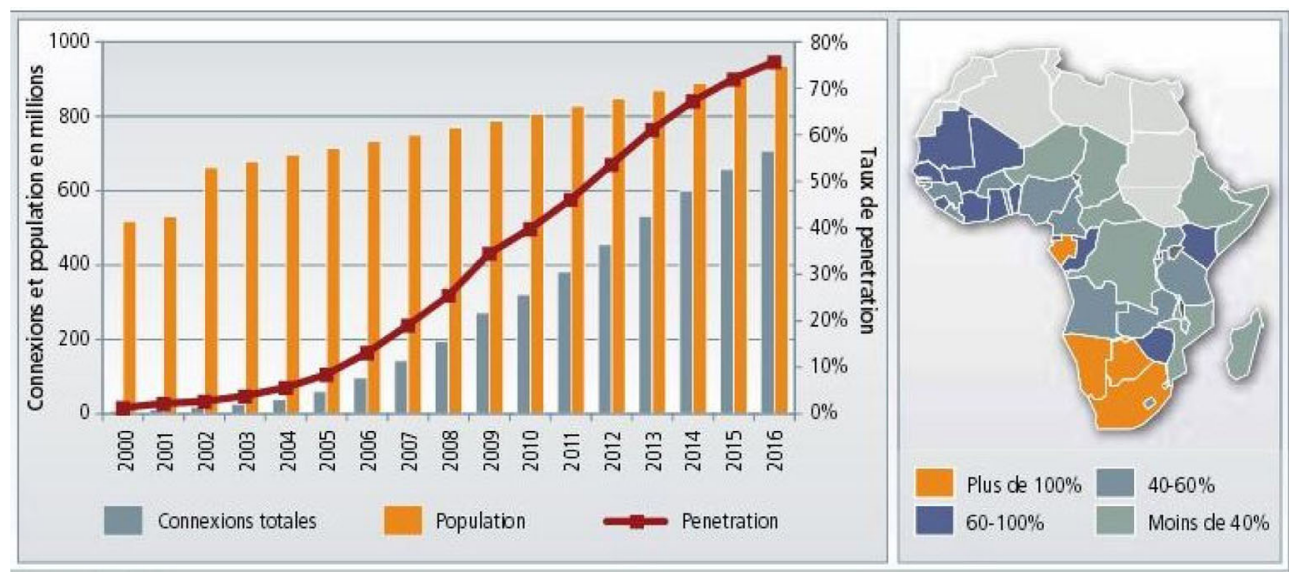

Fig. 5 Nombre de connexions en fonction de la population, taux de pénétration et évolution de la téléphonie mobile en Afrique subsaharienne de 2000 à 2016

Source : Groupe Speciale Mobile Association (GSMA) Wireless intelligence et [37]

prévention, elle concerne les risques avérés dont la fréquence de survenue est inconnue. L'incertitude n'est que sur la réalisation du risque et non sur le risque lui-même. Dans le cas de la prudence, les risques ciblés sont aussi avérés et la fréquence de survenue peut être estimée [42]. Néanmoins, il restera toujours les risques qui ne sont pris en charge ni par la prévention ni par la précaution ou la prudence. Ce sont les risques complètement ignorés. Ceux que l'état actuel des connaissances ne permet pas de connaître. Ils ne sont donc connus qu'à posteriori par une découverte scientifique ou par une révélation incidentelle. C'est la part irréductible de l'aléa. Celle qui reste quand on a déjà éliminé le risque calculable, programmable, gérable, probable ou soupçonnable. C'est le risque résiduel, celui qui persiste après toutes les évaluations et anticipations possibles. Dans tous les cas, le risque, mineur ou majeur, est toujours une contingence non souhaitée. Il peut être pris si les bénéfices espérés l'emportent sur les inconvénients compris dans l'accomplissement d'un acte.

Finalement, la vigilance suivie assurée pour les produits pharmacologiques, les biomatériaux, même après leur mise sur le marché, doit être appliquée pour tous produits susceptibles de nocivité pour la santé. C'est la voie à suivre en Afrique aussi même si le chemin paraît encore lointain. Le nouveau contrat social, quelque soit la latitude à travers la planète, fera certainement l'apologie d'une science et d'une technologie innovantes et conscientes c'est-à-dire celles qui intégreront des exigences de santé publique et environnementale.

Remerciements L'auteur adresse ses remerciements aux Drs. Jean-Loup Rey et Jean-Marie Milleliri du Groupe d'intervention en santé publique \& épidémiologie (GISPE, Marseille, France) et à Mme N'Dèye Marème Kâ, Docteur en chirurgie dentaire, spécialiste de prévention et d'hygiène en établissements de santé (District sanitaire de Louga, Sénégal).
Il tient également à remercier chaleureusement toute l'équipe de Springer-Verlag France (Guido Zosimo Landolfo, Nathalie Huilleret, Nathalie L'Horset-Poulain, Marie-Elia Come-Garry...) avec qui il a travaillé avec beaucoup d'enthousiasme depuis le lancement de cette revue en 2009 et ce avec un très grand professionnalisme et une vision novatrice de l'édition médicale et scientifique. À la suite de restructurations réalisées le $1^{\text {er }}$ septembre 2014 , le groupe Lavoisier sera le nouvel éditeur de cette revue.

Liens d'intérêt : A. Ly déclare ne pas avoir de liens d'intérêts.

\section{Réfèrences}

1. Kundi M (2009) The Controversy about a Possible Relationship between Mobile Phone Use and Cancer. Environ Health Perspect 117:316-24

2. Leszczynski D, Xu Z (2010) Mobile phone radiation health risk controversy: the reliability and sufficiency of science behind the safety standards. Health Res Policy Syst 8:2

3. Baan R, Grosse Y[REMOVED HYPERLINK FIELD], LaubySecretan B, [REMOVED HYPERLINK FIELD] et al (2011) Carcinogenicity of radiofrequency electromagnetic fields. Lancet Oncol 12:624-6

4. Carlos Konlack TB, Tchjuidjan R (2011) Analyse de l'impact des ondes électromagnétiques sur l'homme. Afrique Science 7:1-14

5. Samet JM (2011) Radiation and cancer risk: a continuing challenge for epidemiologists. Environ Health 10(Suppl 1):S4

6. Hertz H (1893) Electric waves. Translated by D.E. Jones, London Macmillan co (1893) and New York Dover (1962)

7. Ndagijimana F, Gaudaire F (2013) Au cœur des Ondes/les champs électromagnétiques en question. Dunod, Paris collection UniverSciences

8. WHO : Que sont les champs électromagnétiques ? http://www. who.int/peh-emf/about/WhatisEMF/fr/ (consulté le 27 août 2014)

9. Consales C, Merla C, Marino C, Benassi B (2012) Electromagnetic Fields, Oxidative Stress, and Neurodegeneration. Int J Cell Biol 683897 
10. Osepchuk JM (1983) Biological effects of electromagnetic radiation. John Wiley \& Sons, INC., New York

11. Nobuyuki Hamada, Yuki Fujimichi (2014) Classification of radiation effects for dose limitation purposes: history, current situation and future prospects. J Radiat Res 55:629-40

12. Mironava T, Hadjiargyrou M, Simon M, Rafailovich MH (2012) The Effects of UV Emission from Compact Fluorescent Light Exposure on Human Dermal Fibroblasts and Keratinocytes In Vitro. Photochem Photobiol 88:1497-506

13. Focke F, Schuermann D, Kuster N, Schär P (2009) DNA fragmentation in human fibroblasts under extremely low frequency electromagnetic field exposure. Mutation Research 683:74-83

14. Wakeford R (2009) Radiation in the workplace-a review of studies of the risks of occupational exposure to ionising radiation. J Radiol Prot 29:A61-79

15. Baskar R, Lee KA, Yeo R, Yeoh KW (2012) Cancer and Radiation Therapy: Current Advances and Future Directions. Int J Med Sci 9:193-9

16. Newhauser WD, Durante M (2011) Assessing the risk of second malignancies after modern radiotherapy. Nat Rev Cancer 11:438-48

16. a. Pearce MS, Salotti JA, Little MP, et al (2012) Radiation exposure from CT scans in childhood and subsequent risk of leukaemia and brain tumours: a retrospective cohort study. Lancet 380:499-505

17. Zimmerman JW, Jimenez H, Pennison MJ, et al (2013) Targeted treatment of cancer with radiofrequency electromagnetic fields amplitude-modulated at tumor-specific frequencies Chin J Cancer 32:573-81

18. Ahlbom A, Feychting M, Green A, et al, (2009) ICNIRP (International Commission for Non-Ionizing Radiation Protection) Standing Committee on Epidemiology. Epidemiologic evidence on mobile phones and tumor risk: a review. Epidemiology 20:639-52

19. CIRC (2011) Le CIRC classe les champs électromagnétiques de radiofréquences comme « Peut-être cancérogène pour l'Homme », communiqué de presse 208 du 31 mai 2011 http://www.iarc.fr/fr/ media-centre/pr/2011/pdfs/pr208_F.pdf

20. IARC (2002) Monographs on the evaluation of carcinogenic risks to humans. Non-Ionizing Radiation, Part 1: Static and Extremely Low-Frequency (ELF). Electric and Magnetic Fields 429 pages, http://monographs.iarc.fr/ENG/Monographs/vol80/index.php

20. a. Health Organization (2007) Extremely Low Frequency Fields Environmental Health Criteria Monograph No.238 http://www. who.int/peh-emf/publications/elf_ehc/en/(consulté le 25 août 2014)

21. Coureau G, Bouvier[REMOVED HYPERLINK FIELD] G, Lebailly P, et al (2013) Mobile phone use and brain tumours in the CERENAT case-control study. Occup Environ Med doi: 10.1136/oemed-101754

22. The Interphone Study Group (2010) Brain tumour risk in relation to mobile telephone use: results of the INTERPHONE international case-control study. Int $\mathrm{J}$ Epidemiol 39:675-94 [REMOVED HYPERLINK FIELD]

23. The Interphone Study Group (2011) Acoustic neuroma risk in relation to mobile telephone use: results of the Interphone international case-control study. Cancer Epidemiol 35:453-64

24. Hardell L, Carlberg M, Söderqvist F, Mild KH (2008) Metaanalysis of long-term mobile phone use and the association with brain tumours. Int J Oncol 32:1097-103

25. Hardell L, Carlberg M, Söderqvist F, Mild KH (2013) Casecontrol study of the association between malignant brain tumours diagnosed between 2007 and 2009 and mobile and cordless phone use. Int J Oncol 43:1833-45

26. Han YY, Kano H, Davis DL, et al (2009) Cell phone use and acoustic neuroma: the need for standardized questionnaires and access to industry data. Surg Neurol 72:216-22

27. Lahkola A, Salminen T, Raitanen J, et al (2008) Meningioma and mobile phone use-a collaborative case-control study in five North European countries. Int J Epidemiol 37:1304-13

28. Moon IS, Kim BG, Kim J, Lee JD, Lee WS (2014) Association between vestibular schwannomas and mobile phone use. Tumour Biol 35:581-7

29. Aydin D, Feychting M, Schüz J, et al (2011) Mobile phone use and brain tumors in children and adolescents: a multicenter casecontrol study. J Natl Cancer Inst 103:1264-76

30. Omuro A, DeAngelis LM (2013) Glioblastoma and other malignant gliomas, a clinical review. JAMA 310:1842-50

31. Ferlay J, Soerjomataram I, Ervik M, et al (2012) Cancer Incidence and Mortality Worldwide. IARC CancerBase

31. a. Ly A (2014) Risques biologiques et risques non biologiques : complexité des déterminants du cancer en Afrique, $\mathrm{XX}^{\mathrm{e}}$ Actualités du Pharo « les maladies non transmissibles en milieu tropical» Groupe d'intervention en Santé Publique et Epidémiologie (GISPE), Marseille, 11-12 septembre 2014, p. 22

32. Sanson M, Wei Wang X, Boisselier B, et al (2010) Mutation des gènes IDH1/IDH2 : un nouveau marqueur pronostique dans les gliomes. Rev Neurol 166(S1): 22-3

33. Wei XB, Jin TB, Li G, et al (2014) CCDC26 Gene Polymorphism and Glioblastoma Risk in the Han Chinese Population Asian Pacific. J Cancer Prev15:3629-33

34. Shete S, Hosking FJ, Robertson LB, et al (2009) Genome-wide association study identifies five susceptibility loci for glioma. Nat Genet 1:899-904

35. Wrensch M, Jenkins RB, Chang JS, et al (2009) Variants in the CDKN2B and RTEL1 regions are associated with high-grade glioma susceptibility. Nat Genet 41:905-8

36. Valdés-Valdivieso L, Penteriani G (2012) Rapport 2012 de Observatoire de la téléphonie mobile en Afrique subsaharienne GSMA et Deloitte, Novembre 2012 http://www.gsma.com/publicpolicy/ wpcontent/uploads/2013/04/gsma_ssa_obs_exec_french_web_01_ 13.pdf (consulté le 27 août 2014)

37. Le boom de la téléphonie mobile en Afrique. http://www.senecoplus.com/technologie-le-grand-boom-de-la-telephonie-mobile-enafrique/ (consulté le 27 août 2014)

38. Pisani F (2013) Le portable révolutionne l'Afrique http://www. lemonde.fr/idees/article/2013/08/18/le-portable-revolutionne afrique_3463006_3232.html (consulté le 27 août 2014)

39. Liang XJ, Kumar A, Shi D, Cui D (2012) Nanostructures for Medicine and Pharmaceuticals. J Nanomaterials doi.org/10.1155/ 2012/921897

40. Atlan H (1999) La fin du tout génétique ? Vers de nouveaux paradigmes en biologie. INRA Éditions (coll. Sciences en questions)

41. Kahn A (1996) Société et révolution biologique. Pour une éthique de la responsabilité. INRA Éditions (coll Sciences en questions)

42. Hunyadi M (2004) Les usages de la précaution. Préface de JeanPierre Dupuy, Genève, Droz, 2004

43. http://b4tea.com/food-health/glioblastoma-multiforme-symptomsdiagnosis-treatment/

44. http://www.edimark.fr/phototheque/galerie_detail.php?id_galerie= 917 